LAPTH-058/13

\title{
Bethe vectors of quantum integrable models based on $U_{q}\left(\widehat{\mathfrak{g l}}_{N}\right)$
}

\author{
S. Pakuliak ${ }^{a}$, E. Ragoucy ${ }^{b}$, N. A. Slavnov ${ }^{*}$ \\ ${ }^{a}$ Laboratory of Theoretical Physics, JINR, 141980 Dubna, Moscow reg., Russia, \\ Moscow Institute of Physics and Technology, 141700, Dolgoprudny, Moscow reg., Russia, \\ Institute of Theoretical and Experimental Physics, 117259 Moscow, Russia \\ ${ }^{b}$ Laboratoire de Physique Théorique LAPTH, CNRS and Université de Savoie, \\ BP 110, 74941 Annecy-le-Vieux Cedex, France \\ ${ }^{c}$ Steklov Mathematical Institute, Moscow, Russia
}

\begin{abstract}
We study quantum $U_{q}\left(\widehat{\mathfrak{g l}}_{N}\right)$ integrable models solvable by the nested algebraic Bethe ansatz. Different formulas are given for the right and left universal off-shell nested Bethe vectors. It is shown that these formulas can be related by certain morphisms of the positive Borel subalgebra in $U_{q}\left(\widehat{\mathfrak{g l}}_{N}\right)$ into analogous subalgebra in $U_{q^{-1}}\left(\widehat{\mathfrak{g l}}_{N}\right)$.
\end{abstract}

*pakuliak@theor.jinr.ru, eric.ragoucy@lapp.in2p3.fr, nslavnov@mi.ras.ru 


\section{Introduction}

The nested algebraic Bethe ansatz [1, 2, 3] in its original formulation allows one to get the Bethe equations as conditions that the Bethe vectors (BV) are eigenstates of the transfer matrix. Nevertheless, even when the Bethe parameters are free and do not satisfy any restriction, the structure of the BV (sometimes such BV are called off-shell) is rather complicated. In the theory of solutions of the quantum Knizhnik-Zamolodchikov equation [4] the universal off-shell $\mathrm{BV}$ were given by certain trace over auxiliary spaces of the products of the monodromy matrices and R-matrices. This presentation allows one to investigate the structure of the nested off-shell BV. It also leads to the explicit formulas for the nested BV when the quantum space of the integrable model becomes the tensor product of evaluation representations of the Yangian or of the Borel subalgebra of the quantum affine algebra $U_{q}\left(\widehat{\mathfrak{g l}}_{N}\right)$ [5].

The explicit formulas for the off-shell BV in terms of the matrix elements of the monodromy matrix were obtained in the papers [6, 7] in the framework of the so-called current approach. In this method the off-shell BV are identified with projections of the product of $U_{q}\left(\widehat{\mathfrak{g l}}_{N}\right)$ currents onto intersections of the standard and current Borel subalgebras in the quantum affine algebra $U_{q}\left(\widehat{\mathfrak{g l}}_{N}\right)$. The theory of these projections was elaborated in the pioneering paper 8 and then fully developed in [9].

The main results of the paper [6] is a development of the method firstly discovered in [10] to calculate the projections of the product of the currents in the case of quantum affine algebra $U_{q}\left(\widehat{\mathfrak{g l}}_{N}\right)$. It was shown in [7] that in order to obtain different presentations of the BV associated with two different embeddings $U_{q}\left(\widehat{\mathfrak{g l}}_{N-1}\right)$ into $U_{q}\left(\widehat{\mathfrak{g l}}_{N}\right)$, one needs to explore two different current realizations of the quantum affine algebra $U_{q}\left(\widehat{\mathfrak{g l}}_{N}\right)$. It becomes clear now that these two different current realizations are related by certain morphisms. In the present paper we show, in particular, how these maps allow us to obtain the dual (or left) off-shell BV from the right ones.

It was shown in [1, 2, 3] that the spectrum of the transfer matrix can be obtained without the use of explicit formulas for BV. Such explicit formulas, however, are very important for the calculation of scalar products of $\mathrm{BV}$, which, in turn, are the main tools for the analysis of correlation functions and form factors of local operators in the Bethe ansatz solvable models. To address this very complicated problem in the case of integrable models with $U_{q}\left(\widehat{\mathfrak{g l}}_{N}\right)$ symmetry, one has to get convenient formulas for the off-shell BV. Such type of presentations were obtained in [11] for the $\mathrm{BV}$ in the models with $\mathfrak{g l}_{3}$-invariant $\mathrm{R}$-matrix. There the explicit formulas for off-shell BV were given in terms of sums over partitions of the sets of Bethe parameters. These expressions also include the Izergin determinant [12], which is the partition function of the six-vertex model with domain wall boundary conditions [13]. The properties of the Izergin determinant allow one to obtain compact formulas for the scalar products of BV in several important particular cases. Using these formulas and the solution of the inverse scattering problem [14, 15] we succeeded to calculate form factors of some local operators in the SU(3)invariant $X X X$ Heisenberg chain [16].

The main goal of this paper is to extend the results of [11] for the models with $U_{q}\left(\widehat{\mathfrak{g l}}_{N}\right)$ symmetry. Our starting point is the explicit formulas for the off-shell nested BV obtained in [6, 7] in terms of the summation over permutations of the whole set of Bethe parameters. 
First we observe that partial summations over permutations give the Izergin determinants. The remaining symmetrization then leads to explicit formulas for the right and left off-shell BV in terms of sums over partitions of the sets of Bethe parameters. We will obtain two different presentations for the same right BV corresponding to the different ways of embedding $U_{q}\left(\widehat{\mathfrak{g l}}_{N-1}\right)$ into $U_{q}\left(\widehat{\mathfrak{g l}}_{N}\right)$.

Then using special properties of the trigonometric R-matrix, we will define the special morphism of $U_{q}\left(\widehat{\mathfrak{g l}}_{N}\right)$ to $U_{q^{-1}}\left(\widehat{\mathfrak{g l}}_{N}\right)$ and prove that different presentations for the right off-shell

$\mathrm{BV}$ can be related by this morphism. Further we will define one more antimorphism of $U_{q}\left(\widehat{\mathfrak{g l}}_{N}\right)$ to $U_{q^{-1}}\left(\widehat{\mathfrak{g l}}_{N}\right)$ which allows us to obtain the formulas in terms of sums over partitions for the left (or dual) off-shell BV.

\section{$1.1 \quad$ Notations}

In this paper we consider the quantum integrable models defined by the $N \times N$ monodromy matrix $T_{i j}(z)$ satisfying the commutation relation

$$
\mathrm{R}(u, v ; q) \cdot(T(u) \otimes \mathbf{1}) \cdot(\mathbf{1} \otimes T(v))=(\mathbf{1} \otimes T(v)) \cdot(T(u) \otimes \mathbf{1}) \cdot \mathrm{R}(u, v ; q),
$$

where $\mathrm{R}(u, v ; q) \in \operatorname{End}\left(\mathbb{C}^{N} \otimes \mathbb{C}^{N}\right) \otimes \mathbb{C}[[v / u]]$, is a trigonometric R-matrix associated with the vector representation of $U_{q}\left(\widehat{\mathfrak{g l}}_{N}\right)$. Here $q$ is a complex parameter neither equal to zero nor root of unity. The algebra (1.1) describes also the commutation relation in the standard Borel subalgebra of the quantum affine algebra $U_{q}\left(\widehat{\mathfrak{g l}}_{N}\right)$. In what follows we will describe the morphisms of this subalgebra of $U_{q}\left(\widehat{\mathfrak{g l}}_{N}\right)$ into the analogous subalgebra of $U_{q^{-1}}\left(\widehat{\mathfrak{g l}}_{N}\right)$ and this is a reason why we are writing the explicit dependence of the R-matrix on the parameter $q$.

More explicitly this R-matrix can be written in the form

$$
\begin{array}{r}
\mathrm{R}(u, v ; q)=\mathrm{f}_{q}(u, v) \\
\sum_{1 \leq i \leq N} \mathrm{E}_{i i} \otimes \mathrm{E}_{i i}+\sum_{1 \leq i<j \leq N}\left(\mathrm{E}_{i i} \otimes \mathrm{E}_{j j}+\mathrm{E}_{j j} \otimes \mathrm{E}_{i i}\right) \\
+\sum_{1 \leq i<j \leq N}\left(\mathrm{~g}_{q}^{(l)}(u, v) \mathrm{E}_{i j} \otimes \mathrm{E}_{j i}+\mathrm{g}_{q}^{(r)}(u, v) \mathrm{E}_{j i} \otimes \mathrm{E}_{i j}\right),
\end{array}
$$

where $\left(\mathrm{E}_{i j}\right)_{l k}=\delta_{i l} \delta_{j k}, i, j, l, k=1, \ldots, N$ are $N \times N$ matrices with unit in the intersection of $i$ th row and $j$ th column and zero elsewhere, and the coefficient functions are defined as follow 1

$$
\mathrm{f}_{q}(u, v)=\frac{q u-q^{-1} v}{u-v}, \quad \mathrm{~g}_{q}(u, v)=\frac{\left(q-q^{-1}\right)}{u-v}
$$

and

$$
\mathrm{g}_{q}^{(l)}(u, v)=u \mathrm{~g}_{q}(u, v), \quad \mathrm{g}_{q}^{(r)}(u, v)=v \mathrm{~g}_{q}(u, v) .
$$

A concrete quantum integrable model is defined usually by a certain representation space $V$ (or quantum space of the integrable models) where the entries of the monodromy matrix $T_{i, j}(u)$

\footnotetext{
${ }^{1}$ When there is no ambiguity, we will omit the subscript $q$ in the rational functions (1.3) to simplify the formulas.
} 
act. The space of states for this model is identified with a representation space of the Borel subalgebra of $U_{q}\left(\widehat{\mathfrak{g l}}_{N}\right)$ generated by the vector $|0\rangle$ satisfying the following conditions

$$
T_{j, i}(z)|0\rangle=0, \quad j>i, \quad T_{i, i}(z)|0\rangle=\lambda_{i}(z)|0\rangle, \quad i=1, \ldots, N .
$$

The functions $\lambda_{i}(z)$ characterize the concrete integrable model and the vector $|0\rangle$ is a common eigenvector of the diagonal entries of the monodromy matrix. Although the diagonal matrix elements do not commute, such a vector exists because the commutators $\left[T_{i, i}(z), T_{j, j}\left(z^{\prime}\right)\right], \forall i, j$, annihilate the vacuum vector $|0\rangle$, due to the commutation relations and (1.5).

The off-shell BV are constructed as special polynomials of the monodromy matrix elements $T_{i, j}(z), i \leq j$, depending on sets of parameters (the Bethe parameters) and acting on $|0\rangle$. These parameters are supposed to be generic complex numbers. If they satisfy the system of the nested Bethe ansatz equations, then the corresponding BV becomes an eigenvector of the transfer matrix 2 .

We will also consider the dual (or left) off-shell BV, which belong to the dual space $V^{*}$. They are generated by the matrix elements $T_{i, j}(z)$ acting on a vector $\langle 0|$, which satisfies the conditions

$$
\langle 0| T_{i, j}(z)=0, \quad j>i, \quad\langle 0| T_{i, i}(z)=\lambda_{i}(z)\langle 0|, \quad i=1, \ldots, N .
$$

\section{Formulas for BV from the current approach}

The goal of this section is to remind the formulas for the right off-shell BV formulated by the Theorem 2 of the paper [7] in the form of sums over all permutations of the Bethe parameters of the same sort.

Let $\bar{n}=\left\{n_{1}, n_{2}, \ldots, n_{N-1}\right\}$ be a collection of positive integers and let $\bar{t}_{\bar{n}}$ be a set of variables

$$
\bar{t}_{\bar{n}}=\left\{t_{1}^{1}, \ldots, t_{n_{1}}^{1} ; t_{1}^{2}, \ldots, t_{n_{2}}^{2} ; \ldots \ldots ; t_{1}^{N-2}, \ldots, t_{n_{N-2}}^{N-2} ; t_{1}^{N-1}, \ldots, t_{n_{N-1}}^{N-1}\right\} .
$$

Here superscripts indicate the type of Bethe parameter and correspond to the simple roots of the algebra $\mathfrak{g l}_{N}$. There are $N-1$ different sorts of Bethe parameters in the generic BV for $U_{q}\left(\widehat{\mathfrak{g l}}_{N}\right)$-integrable model. The subscript counts the number of the Bethe parameters of the same type. For a generic BV, we denote by $n_{i}$ the total number of type $i$ Bethe parameters.

Let us consider a direct product of the symmetric groups: $S_{\bar{n}}=S_{n_{1}} \times \cdots \times S_{n_{N-1}}$. For any function $G\left(\bar{t}_{\bar{n}}\right)$ we denote by

$$
\operatorname{Sym}_{\bar{t}_{\bar{n}}} G\left(\bar{t}_{\bar{n}}\right)=\sum_{\sigma \in S_{\bar{n}}} G\left({ }^{\sigma} \bar{t}_{\bar{n}}\right), \quad \sigma=\left\{\sigma^{1}, \sigma^{2}, \ldots, \sigma^{N-1}\right\}
$$

a symmetrization over groups of variables of same type $k,\left\{t_{1}^{k}, \ldots, t_{n_{k}}^{k}\right\}$, where

$$
\sigma \bar{t}_{\bar{n}}=\left\{t_{\sigma^{1}(1)}^{1}, \ldots, t_{\sigma^{1}\left(n_{1}\right)}^{1} ; \ldots ; t_{\sigma^{N-1}(1)}^{N-1}, \ldots, t_{\sigma^{N-1}\left(n_{N-1}\right)}^{N-1}\right\} .
$$

Let

$$
\beta\left(\bar{t}_{\bar{n}}\right)=\prod_{k=1}^{N-1} \prod_{1 \leq \ell<\ell^{\prime} \leq n_{k}} \mathrm{f}\left(t_{\ell^{\prime}}^{k}, t_{\ell}^{k}\right)
$$

\footnotetext{
${ }^{2}$ Sometimes such vectors are called on-shell BV.
} 
be a function of the formal variables $t_{\ell}^{k}, \ell=1, . ., n_{k}, k=1, \ldots, N-1$.

In order to describe formulas for the off-shell BV we need the following combinatorial data. Let $[\bar{m}]=\left\{m_{j}^{i}\right\}$ and $[\bar{s}]=\left\{s_{i}^{j}\right\}$ for $1 \leq i \leq j \leq N-1$ be two collections of the nonnegative integers. We say that collections $[\bar{m}]$ and $[\bar{s}]$ are $\bar{n}$ upper or lower (resp.) permissible, if they satisfy the following conditions

$$
m_{i}^{i} \geq m_{i+1}^{i} \geq \cdots \geq m_{N-1}^{i} \geq m_{N}^{i}=0, \quad n_{i}=\sum_{j=1}^{i} m_{i}^{j}, \quad i=1, \ldots, N-1,
$$

and

$$
0=s_{0}^{i} \leq s_{1}^{i} \leq \cdots \leq s_{i-1}^{i-1} \leq s_{i}^{i}, \quad n_{i}=\sum_{j=i}^{N-1} s_{i}^{j}, \quad i=1, \ldots, N-1,
$$

respectively. We also follow the convention $m_{N}^{j}=s_{0}^{j}=0$ for $j=1, \ldots, N-1$.

The collections of upper or lower permissible integers $[\bar{m}]$ and $[\bar{s}]$ can be visualized as upper or lower triangular matrices

$$
[\bar{m}]=\left(\begin{array}{ccccc}
m_{1}^{1} & m_{2}^{1} & \ldots & m_{N-2}^{1} & m_{N-1}^{1} \\
0 & m_{2}^{2} & \ldots & m_{N-2}^{2} & m_{N-1}^{2} \\
& & \ddots & \vdots & \vdots \\
& 0 & & m_{N-2}^{N-2} & m_{N-1}^{N-2} \\
& & & & m_{N-1}^{N-1}
\end{array}\right) \quad \begin{gathered}
0=m_{N}^{1} \leq m_{N-1}^{1} \leq \ldots \leq m_{1}^{1} \\
0=m_{N}^{2} \leq m_{N-1}^{2} \leq \ldots \leq m_{2}^{2} \\
0=m_{N}^{N-2} \leq m_{N-1}^{N-2} \leq m_{N-2}^{N-2} \\
0=m_{N}^{N-1} \leq m_{N-1}^{N-1}
\end{gathered}
$$

and

$$
[\bar{s}]=\left(\begin{array}{ccccc}
s_{1}^{1} & & & & \\
s_{1}^{2} & s_{2}^{2} & & 0 & \\
\vdots & \vdots & \ddots & & \\
s_{1}^{N-2} & s_{2}^{N-2} & \ldots & s_{N-2}^{N-2} & \\
s_{1}^{N-1} & s_{2}^{N-1} & \ldots & s_{N-2}^{N-1} & s_{N-1}^{N-1}
\end{array}\right) \begin{gathered}
0=s_{0}^{1} \leq s_{1}^{1} \\
0=s_{0}^{2} \leq s_{1}^{2} \leq s_{2}^{2} \\
\vdots \\
0=s_{0}^{N-1} \leq s_{1}^{N-1} \leq \ldots \leq s_{N-1}^{N-1}
\end{gathered}
$$

In words, $[\bar{m}]$ is an upper triangular matrix with integer entries, ordered on each lines, and such that the sum of its $i^{t h}$ column gives back $n_{i}$. In what follows, $[\bar{m}]$ (resp. $[\bar{s}]$ ) will denote upper (resp. lower) permissible collections of integers.

Let $\bar{m}^{j}$ and $\bar{s}^{j}, j=1, \ldots, N-1$ be the $j$-th rows of the permissible matrices $[\bar{m}]$ and $[\bar{s}]$. Define a collection of vectors

$$
\begin{gathered}
\overline{\mathbf{m}}^{j}=\bar{m}^{1}+\bar{m}^{2}+\cdots+\bar{m}^{j-1}+\bar{m}^{j}, \quad j=1, \ldots, N-1, \\
\overline{\mathbf{s}}^{j}=\bar{s}^{j}+\bar{s}^{j+1}+\cdots+\bar{s}^{N-2}+\bar{s}^{N-1}, \quad j=1, \ldots, N-1,
\end{gathered}
$$


with non-negative integer components. Set $\overline{\mathbf{m}}^{0}=\overline{0}$ and $\overline{\mathbf{s}}^{N}=\overline{0}$. Denote by $\mathbf{m}_{a}^{j}$ and $\mathbf{s}_{a}^{j}$ the components of the vectors $\overline{\mathbf{m}}^{j}$ and $\overline{\mathbf{s}}^{j}$ :

$$
\begin{aligned}
\overline{\mathbf{m}}^{j} & =\left\{n_{1}, n_{2}, \ldots, n_{j}, m_{j+1}^{1}+\cdots+m_{j+1}^{j}, \ldots, m_{N-1}^{1}+\cdots+m_{N-1}^{j}\right\}, \\
\overline{\mathbf{s}}^{j} & =\left\{s_{1}^{j}+\cdots+s_{1}^{N-1}, \ldots, s_{j-1}^{j}+\cdots+s_{j-1}^{N-1}, n_{j}, \ldots, n_{N-2}, n_{N-1}\right\} .
\end{aligned}
$$

According to the conditions (2.5) and (2.4), $\overline{\mathbf{m}}^{N-1}=\overline{\mathbf{s}}^{1}=\bar{n}$.

We introduce the following combinations of the $U_{q}\left(\widehat{\mathfrak{g l}}_{N}\right)$ monodromy matrix elements, that we call pre-BV:

$$
\begin{aligned}
\mathcal{B}^{\bar{n}}\left(\bar{t}_{\bar{n}}\right) & =\sum_{[\bar{m}]} \operatorname{Sym}_{\bar{t}_{\bar{n}}}\left(\beta\left(\bar{t}_{\bar{n}}\right) \prod_{1 \leq j \leq i<N}\left(\left[\left(m_{i}^{j}-m_{i+1}^{j}\right) !\right]^{-1} \prod_{n_{j}-m_{i}^{j}<\ell^{\prime}<\ell \leq n_{j}-m_{i+1}^{j}} \mathrm{f}\left(t_{\ell}^{j}, t_{\ell^{\prime}}^{j}\right)^{-1}\right)\right. \\
& \times \prod_{i=2}^{N-1}\left(\prod_{j=1}^{i-1}\left(\prod_{\ell=0}^{m_{i}^{j}-1} \mathrm{~g}^{(l)}\left(t_{\mathbf{m}_{i}^{j}-\ell^{j}}, t_{\mathbf{m}_{i-1}^{j}-\ell^{j}}\right) \prod_{\ell^{\prime}=\mathbf{m}_{i-1}^{j}-\ell+1}^{n_{i-1}} \mathrm{f}\left(t_{\mathbf{m}_{i}^{j}-\ell^{j}}, t_{\ell^{\prime}}^{i-1}\right)\right)\right) \\
& \left.\times \prod_{1 \leq j \leq N-1}^{\longrightarrow}\left(\prod_{N-1 \geq i \geq j}\left(\prod_{\ell=n_{j}-m_{i}^{j}+1}^{n_{j}-m_{i+1}^{j}} T_{j, i+1}\left(t_{\ell}^{j}\right)\right)\right) \prod_{j=1}^{N-1} \prod_{\ell=1}^{n_{j}-m_{j}^{j}} T_{j, j}\left(t_{\ell}^{j}\right)\right),
\end{aligned}
$$

and

$$
\begin{aligned}
& \widehat{\mathcal{B}}^{\bar{n}}\left(\bar{t}_{\bar{n}}\right)=\sum_{[\bar{s}]} \operatorname{Sym}_{\bar{t}_{\bar{n}}}\left(\beta\left(\bar{t}_{\bar{n}}\right) \prod_{1 \leq i \leq j<N}\left(\left[\left(s_{i}^{j}-s_{i-1}^{j}\right) !\right]^{-1} \prod_{s_{i-1}^{j}<\ell^{\prime}<\ell \leq s_{i}^{j}} \mathrm{f}\left(t_{\ell}^{j}, t_{\ell^{\prime}}^{j}\right)^{-1}\right)\right. \\
& \times \prod_{j=2}^{N-1}\left(\prod_{i=1}^{j-1}\left(\prod_{\ell=1}^{s_{i}^{j}} \mathrm{~g}^{(r)}\left(t_{n_{i+1}-\mathbf{s}_{i+1}^{j}+\ell^{i+1}}, t_{n_{i}-\mathbf{s}_{i}^{j}+\ell}\right) \prod_{\ell^{\prime}=1}^{n_{i+1}-\mathbf{s}_{i+1}^{j}+\ell-1} \mathrm{f}\left(t_{\ell^{\prime}}^{i+1}, t_{n_{i}-\mathbf{s}_{i}^{j}+\ell}^{i}\right)\right)\right) \\
& \left.\left.\times \underset{N-1 \geq j \geq 1}{\overleftarrow{\prod_{1 \leq i \leq j}}}\left(\prod_{\ell=s_{i-1}^{j}+1}^{s_{i}^{j}} T_{i, j+1}\left(t_{\ell}^{j}\right)\right)\right) \prod_{j=1}^{N-1} \prod_{\ell=s_{j}^{j}}^{n_{j}} T_{j+1, j+1}\left(t_{\ell}^{j}\right)\right)
\end{aligned}
$$

where the ordered products of the non-commuting entries $A_{i}$ are defined as follows

$$
\overleftarrow{\prod_{i}} A_{i}=A_{n} A_{n-1} \cdots A_{2} A_{1} \text { and } \quad \prod_{i} A_{i}=A_{1} A_{2} \cdots A_{n-1} A_{n}
$$

Theorem 2 in [7] states that the two pre-BVs produce two different presentations of the same off-shell BV:

$$
\mathbb{B}^{\bar{n}}\left(\bar{t}_{\bar{n}}\right)=\mathcal{B}^{\bar{n}}\left(\bar{t}_{\bar{n}}\right)|0\rangle=\widehat{\mathcal{B}}^{\bar{n}}\left(\bar{t}_{\bar{n}}\right)|0\rangle .
$$

The ordering in the products over $\ell$ in the formulas (2.10) and (2.11) is not important, because of the commutativity of the entries of $T$-operators with equal matrix indices. Moreover, the order in the products of the noncommuting diagonal monodromy matrix elements in (2.10) 
and (2.11) is also non-important, since these combinations will eventually act onto the highest weight vector $|0\rangle$ and produce products of scalar functions, due to (1.5).

The formulas (2.10) and (2.11) contain summations over permutations of the symmetric functions and these summations can be calculated. This will be done in the following section and formulas for the pre-BV will be presented as sums over different partitions of the set of Bethe parameters (2.1).

\section{Off-shell BV as sums over partitions}

To save space and simplify formulas, we will use following convention for the products of the commuting entries of the monodromy matrix $T_{i j}$, vacuum eigenvalues $\lambda_{i}$ and functions $\mathrm{f}(u, v)$. Namely, whenever such an operator or a function depends on a set of variables, say $\bar{t}$, this means that we deal with the product of these commuting operators or functions with respect to the corresponding set:

$$
T_{i, j}(\bar{t})=\prod_{t_{k} \in \bar{t}} T_{i, j}\left(t_{k}\right), \quad \lambda_{i}(\bar{t})=\prod_{t_{k} \in \bar{t}} \lambda_{i}\left(t_{k}\right), \quad \mathrm{f}\left(\bar{t}, \bar{t}^{\prime}\right)=\prod_{t_{j} \in \bar{t}} \prod_{t_{k} \in \bar{t}^{\prime}} \mathbf{f}\left(t_{j}, t_{k}\right) .
$$

In various formulas the Izergin determinant $\mathrm{K}_{n}(\bar{x} \mid \bar{y})$ appears [12]. It is defined for two sets $\bar{x}$ and $\bar{y}$ of the same cardinality $\# \bar{x}=\# \bar{y}=n$ :

$$
\mathrm{K}_{n}(\bar{x} \mid \bar{y})=\frac{\prod_{1 \leq i, j \leq k}\left(q x_{i}-q^{-1} y_{j}\right)}{\prod_{1 \leq i<j \leq k}\left(x_{i}-x_{j}\right)\left(y_{j}-y_{i}\right)} \cdot \operatorname{det}\left[\frac{q-q^{-1}}{\left(x_{i}-y_{j}\right)\left(q x_{i}-q^{-1} y_{j}\right)}\right] .
$$

Below we also use two modifications of the Izergin determinant

$$
\mathrm{K}_{n}^{(l)}(\bar{x} \mid \bar{y})=\prod_{i=1}^{n} x_{i} \cdot \mathrm{K}_{n}(\bar{x} \mid \bar{y}), \quad \mathrm{K}_{n}^{(r)}(\bar{x} \mid \bar{y})=\prod_{i=1}^{n} y_{i} \cdot \mathrm{K}_{n}(\bar{x} \mid \bar{y}),
$$

which we call left and right Izergin determinants respectively.

\subsection{Combinatorial description of the partitions}

The main goal of this section is to transform the equations (2.10) and (2.11) for pre-BV into new representations involving sums with respect to partitions of the Bethe parameters. Let us first describe the general strategy of these transforms.

Consider for definiteness (2.10). This representation contains sums of different types. One sum runs over all possible permissible sets $[\bar{m}]$. Each individual term in this sum includes summations over permutations of the Bethe parameters of the same type. For example, the extreme permissible set in (2.10) such that $m_{i}^{j}=\delta_{i}^{j} n_{i}$ corresponds to the term

$$
\frac{1}{n_{1} ! n_{2} ! \cdots n_{N-1} !} \operatorname{Sym}_{\bar{t}_{\bar{n}}}\left(T_{1,2}\left(\bar{t}^{1}\right) T_{2,3}\left(\bar{t}^{2}\right) \cdots T_{N-1, N}\left(\bar{t}^{N-1}\right)\right)
$$

and the summation over permutations of the Bethe parameters can be easily performed due to the commutativity of the entries of the monodromy matrix with the same matrix indices. It is clear that this summation removes the combinatorial factors in (3.4). 
Other terms in the sum over permissible sets in (2.10) can be treated similarly. For any permissible set $[\bar{m}]$ one can consider the sum over permutations of the Bethe parameters of the same type as the sum over special partitions of these parameters into subsets and further permutations within every subset. The partitions of the Bethe parameters into subsets are determined by the permissible set $[\bar{m}]$. They correspond to Bethe parameters that enter the products in (2.10) through the same monodromy matrix entry. Due to the commutativity of such matrix elements the sums over permutations inside these subsets can be calculated via the identities (3.13) and (3.14). They also remove the combinatorial factors. Details of this calculation will be presented below in the proof of the Proposition 3.1 .

The presentation (2.11) can be transformed in the similar manner. As a result we will be left with summations over all possible partitions of the sets of Bethe parameters dictated by the permissible set $[\bar{m}]$ and $[\bar{s}]$. It is convenient to parameterize the subsets of every set $\bar{t}^{k}$ by two positive integers $i$ and $j$ satisfying the conditions

$$
1 \leq i \leq k \leq j \leq N-1, \quad \bar{t}^{k}=\bigcup_{i=1}^{k} \bigcup_{j=k}^{N-1} \bar{t}_{i, j}^{k},
$$

and

$$
\# \bar{t}_{i, j}^{k}=m_{j}^{i}-m_{j+1}^{i} \quad \text { for } \quad i=1, \ldots, k \quad \text { and } \quad j=k, \ldots, N-1, \quad \forall k
$$

for the upper permissible matrix $[\bar{m}]$. For the lower permissible matrix $[\bar{s}]$, we introduce analogous partitions of the same sets (3.5) with similar conditions:

$$
\# \bar{t}_{i, j}^{k}=s_{i}^{j}-s_{i-1}^{j} \quad \text { for } \quad i=1, \ldots, k \quad \text { and } \quad j=k, \ldots, N-1, \quad \forall k .
$$

Note that for any given pair $(i, j)$, we have $\# \bar{t}_{i, j}^{k}=\# \bar{t}_{i, j}^{k^{\prime}}, \forall k, k^{\prime}$. Remark also that, because of the conditions (2.4) and (2.5), we have indeed \# $\# \bar{t}^{k}=\sum_{i=1}^{k} \sum_{j=k}^{N-1} \# \bar{t}_{i, j}^{k}$.

We introduce ordering rules ' $\prec$ ' and ' $\prec^{t}$ ' of these pairs according to the following conventions

$$
i, j \prec i^{\prime}, j^{\prime} \quad \text { if } \quad i<i^{\prime}, \forall j, j^{\prime} \quad \text { or if } i=i^{\prime}, j<j^{\prime},
$$

and

$$
i, j \prec^{t} i^{\prime}, j^{\prime} \quad \text { if } \quad j<j^{\prime}, \quad \forall i, i^{\prime} \quad \text { or if } \quad j=j^{\prime}, i<i^{\prime} .
$$

Example 3.1. To illustrate how combinatorial data encoded into permissible matrices (2.6) and (2.7) is transferred into description of partitions (3.5) we write explicitly the partitions associated with this data in case of the $U_{q}\left(\widehat{\mathfrak{g l}}_{5}\right)$ off-shell $B V$.

In that case, we have 4 types of Bethe parameters $\bar{t}^{1}, \ldots, \bar{t}^{4}$, and the permissible collections lead to $4 \times 4$ triangular matrices of types (2.6) and (2.7). The rules (3.5) show that the sets of Bethe parameters $\bar{t}^{1}$ and $\bar{t}^{4}$ (resp. $\bar{t}^{2}$ and $\bar{t}^{3}$ ) are divided into 4 (resp. 6) subsets. We get the subsets

$$
\begin{aligned}
& \bar{t}^{1}=\bar{t}_{1,1}^{1} \cup \bar{t}_{1,2} \cup \bar{t}_{1,3}^{1} \cup \bar{t}_{1,4}^{1} \\
& \bar{t}^{2}=\quad \bar{t}_{1,2}^{2} \cup \bar{t}_{1,3}^{2} \cup \bar{t}_{1,4}^{2} \cup \bar{t}_{2,2}^{2} \cup \bar{t}_{2,3}^{2} \cup \bar{t}_{2,4}^{2} \\
& \vec{t}^{3}=\quad \vec{t}_{1,3}^{3} \cup \vec{t}_{1,4}^{3} \cup \cup \quad \cup \vec{t}_{2,3}^{3} \cup \vec{t}_{2,4}^{3} \cup \bar{t}_{3,3}^{3} \quad \cup \quad \vec{t}_{3,4}^{3} \\
& \bar{t}^{4}=\quad \bar{t}_{1,4}^{4} \cup \cup \quad \cup \bar{t}_{2,4}^{4} \cup \cup \quad \cup \bar{t}_{3,4}^{4} \cup \bar{t}_{4,4}^{4}
\end{aligned}
$$


for (2.6) and the subsets

$$
\begin{array}{llllllllllllllllllll}
\bar{t}^{4}= & \bar{t}_{4,4}^{4} & \cup & \bar{t}_{3,4}^{4} & \cup & \bar{t}_{2,4}^{4} & \cup & \bar{t}_{1,4}^{4} & & & & & & & & \\
\bar{t}^{3}= & & \bar{t}_{3,4}^{3} & \cup & \bar{t}_{2,4}^{3} & \cup & \bar{t}_{1,4}^{3} & \cup & \bar{t}_{3,3}^{3} & \cup & \bar{t}_{2,3}^{3} & \cup & \bar{t}_{2,4}^{3} & & & & & \\
\bar{t}^{2}= & & & \bar{t}_{2,4}^{2} & \cup & \bar{t}_{1,4}^{2} & \cup & & \cup & \bar{t}_{2,3}^{3} & \cup & \bar{t}_{1,3}^{3} & \cup & \bar{t}_{2,2}^{3} & \cup & \bar{t}_{1,2}^{2} & \\
\bar{t}^{1}= & & & & \bar{t}_{1,4}^{1} & \cup & & & & \cup & \bar{t}_{1,3}^{1} & \cup & & \cup & \bar{t}_{1,2}^{1} & \cup & \bar{t}_{1,1}^{1}
\end{array}
$$

for 2.7). The subsets in the same column have the same cardinality, that is defined by the formulas (3.6) and (3.7).

Now we can formulate the following

Proposition 3.1. The formulas (2.10) and (2.11) for the universal pre-BV can be written as sums over partitions of the Bethe parameters (3.5), (3.6) and (3.7) as follows

$$
\begin{aligned}
\mathcal{B}^{\bar{n}}\left(\bar{t}_{\bar{n}}\right) & =\sum_{\operatorname{part}} \prod_{k=1}^{N-1} \prod_{i, j \prec i^{\prime}, j^{\prime}} \mathrm{f}\left(\bar{t}_{i^{\prime}, j^{\prime}}^{k}, \bar{t}_{i, j}^{k}\right) \prod_{k=2}^{N-1}\left(\prod_{i, j \prec i^{\prime}, j^{\prime}} \mathrm{f}\left(\bar{t}_{i, j}^{k}, \bar{t}_{i^{\prime}, j^{\prime}}^{k-1}\right) \prod_{i<j} \mathrm{~K}^{(l)}\left(\bar{t}_{i, j}^{k} \mid \bar{t}_{i, j}^{k-1}\right)\right) \\
& \times \underset{1 \leq k \leq N-1}{\longrightarrow}\left(\prod_{N \geq j>k} T_{k, j}\left(\bar{t}_{k, j-1}^{k}\right)\right) \prod_{k=2}^{N-1} \prod_{i, j \prec k, k} T_{k, k}\left(\bar{t}_{i, j}^{k}\right),
\end{aligned}
$$

and

$$
\begin{aligned}
\widehat{\mathcal{B}}^{\bar{n}}\left(\bar{t}_{\bar{n}}\right) & =\sum_{\operatorname{part}} \prod_{k=1}^{N-1} \prod_{i, j \prec^{t} i^{\prime}, j^{\prime}} \mathrm{f}\left(\bar{t}_{i^{\prime}, j^{\prime}}^{k}, \bar{t}_{i, j}^{k}\right) \prod_{k=2}^{N-1}\left(\prod_{i, j \prec^{t} i^{\prime}, j^{\prime}} \mathrm{f}\left(\bar{t}_{i, j}^{k}, \bar{t}_{i^{\prime}, j^{\prime}}^{k-1}\right) \prod_{i<j} \mathrm{~K}^{(r)}\left(\bar{t}_{i, j}^{k} \mid \bar{t}_{i, j}^{k-1}\right)\right) \\
& \times \overleftrightarrow{\prod_{N-1 \geq k \geq 1}}\left(\prod_{1 \leq j \leq k}^{\longrightarrow} T_{j, k+1}\left(\bar{t}_{j, k}^{k}\right)\right) \prod_{k=1}^{N-2} \prod_{k, k \prec^{t} i, j} T_{k+1, k+1}\left(\bar{t}_{i, j}^{k}\right) .
\end{aligned}
$$

Proof. The basic idea is to replace the summations over permutations of the Bethe parameters and permissible matrices $[\bar{m}],[\bar{s}]$ in (2.10) and (2.11) by the summations over partitions of the sets of the Bethe parameters. Once it is done, the sum over permutations within any fixed subset can be calculated using certain identities of the rational functions (3.13) and (3.14). To formulate these identities we introduce the following notations. For any sets $\bar{x}$ and $\bar{y}$ with equal cardinalities $\# \bar{x}=\# \bar{y}=n$, we introduce rational functions

$$
\mathrm{B}(\bar{y})=\prod_{\ell<\ell^{\prime}} \mathrm{f}\left(y_{\ell^{\prime}}, y_{\ell}\right), \quad \mathrm{G}(\bar{y} \mid \bar{x})=\prod_{\ell=1}^{n} \mathrm{~g}\left(y_{\ell}, x_{\ell}\right), \quad \mathrm{F}(\bar{y} \mid \bar{x})=\prod_{\ell<\ell^{\prime}} \mathrm{f}\left(y_{\ell}, x_{\ell^{\prime}}\right) .
$$

Then the following identities of the rational functions are valid:

$$
\begin{aligned}
\operatorname{Sym}_{\bar{y}}(\mathrm{~B}(\bar{y}) \mathrm{G}(\bar{y} \mid \bar{x}) \mathrm{F}(\bar{y} \mid \bar{x})) & =\mathrm{K}_{n}(\bar{y} \mid \bar{x}), \\
\operatorname{Sym}_{\bar{x}}(\mathrm{~B}(\bar{x}) \mathrm{G}(\bar{y} \mid \bar{x}) \mathrm{F}(\bar{y} \mid \bar{x})) & =\mathrm{K}_{n}(\bar{y} \mid \bar{x}) .
\end{aligned}
$$


The proof of these identities in the case of quantum integrable models associated with rational R-matrix is given in Appendix A of the paper [19]. The proof in the trigonometric case is completely analogous.

According to the partitions defined by the permissible matrices $[\bar{m}]$ and $[\bar{s}]$ the rational function $\beta\left(\bar{t}_{\bar{n}}\right)$ can be rewritten as

$$
\beta\left(\bar{t}_{\bar{n}}\right)=\prod_{k=1}^{N-1} \prod_{1 \leq \ell<\ell^{\prime} \leq n_{k}} \mathrm{f}\left(t_{\ell^{\prime}}^{k}, t_{\ell}^{k}\right)=\prod_{k=1}^{N-1} \mathrm{~B}\left(\bar{t}^{k}\right)=\prod_{i \leq k \leq j} \mathrm{~B}\left(\bar{t}_{i, j}^{k}\right),
$$

where $\bar{t}_{i, j}^{k}$ are defined either by (3.6) or by (3.7).

We show the equivalence of (2.10) and (3.10) first, using partitions (3.6). We will decompose the proof in the series of steps.

1. The product of rational functions $\prod_{i \leq j}^{N-1} \mathrm{~B}\left(\bar{t}_{i, j}^{i}\right)$ entering the function $\beta\left(\bar{t}_{\bar{n}}\right)$ will be canceled out by the product of the rational functions in the first row of (2.10). This allows to cancel some of the factorial factors. At this step the expression in (2.10) is symmetric with respect to permutations in the set $\bar{t}_{N-1, N-1}^{N-1}$ and the factorial factor $\left(m_{N-1}^{N-1}\right)^{-1}$ disappear.

2. At this step, for $k=1, \ldots, N-2$, we can select the following product of rational functions in the r.h.s. of (2.10)

$$
\mathrm{B}\left(\bar{t}_{k, N-1}^{N-1}\right) \mathrm{G}\left(\bar{t}_{k, N-1}^{N-1} \mid \bar{t}_{k, N-1}^{N-2}\right) \mathrm{F}\left(\bar{t}_{k, N-1}^{N-1} \mid \bar{t}_{k, N-1}^{N-2}\right) .
$$

Since all the other factors in the r.h.s. of (2.10) are symmetric with respect to the permutations in the set $\bar{t}_{k, N-1}^{N-1}$, we can perform the symmetrization over these sets and apply the identity (3.13) to obtain the product over $k$ of the Izergin determinants $\mathrm{K}\left(\bar{t}_{k, N-1}^{N-1} \mid \bar{t}_{k, N-1}^{N-2}\right)$ which are symmetric with respect to permutations in the sets $\bar{t}_{k, N-1}^{N-2}$. Moreover the factorial factors $\left(\left(m_{N-2}^{N-2}-m_{N-1}^{N-2}\right) ! m_{N-1}^{N-2} !\right)^{-1}$ will disappear since the expression under summation is symmetric with respect to permutations in the sets $\bar{t}_{N-2, N-2}^{N-2}$ and $\bar{t}_{N-2, N-1}^{N-2}$.

3. Next we perform an analogous procedure to obtain the product of the Izergin determinants over $k=1, \ldots, N-3$

$$
\mathrm{K}\left(\bar{t}_{k, N-2}^{N-2} \mid \bar{t}_{k, N-2}^{N-3}\right) \mathrm{K}\left(\bar{t}_{k, N-1}^{N-2} \mid \bar{t}_{k, N-1}^{N-3}\right),
$$

which is symmetric with respect to permutations in the sets $\bar{t}_{k, N-1}^{N-3}$ and $\bar{t}_{k, N-2}^{N-3}$. Now the combinatorial factors $\left(\left(m_{N-3}^{N-3}-m_{N-2}^{N-3}\right) !\left(m_{N-2}^{N-3}-m_{N-1}^{N-3}\right) ! m_{N-1}^{N-3} !\right)^{-1}$ will disappear due to the symmetry of the summand with respect to permutations in the sets $\bar{t}_{N-3, N-3}^{N-3}$, $\bar{t}_{N-3, N-2}^{N-3}$ and $\bar{t}_{N-3, N-1}^{N-3}$.

4. We iterate these symmetrizations over the sets up to the final step, that corresponds to the symmetrizations over the sets $\vec{t}_{1, k}^{2}, k=2, \ldots, N-1$. We obtain in this way the product of Izergin determinants $\prod_{k=2}^{N-1} \mathrm{~K}\left(\vec{t}_{1, k}^{2} \mid \vec{t}_{1, k}\right)$ and the last combinatorial factor $\prod_{k=1}^{N-1}\left(\left(m_{k}^{1}-m_{k+1}^{1}\right) !\right)^{-1}$ disappears, due to symmetry arguments. 
Besides the product of Izergin determinants described above, we are also left with the product of rational functions, which can we written as in the first line of (3.10) using the ordering of the sets introduced by (3.8).

The proof of equivalence between (2.11) and (3.11) is analogous. The only difference is that we have to start the whole procedure with the partitions (3.7), and begin with the symmetrizations over sets $\bar{t}_{1,1}^{1}, \bar{t}_{1,2}^{1}$ and so on. Then, we use the identity (3.14) to produce the product of the Izergin determinants.

\section{Morphisms}

The aim of this section is to describe certain morphisms which relate the algebras $U_{q}\left(\widehat{\mathfrak{g l}}_{N}\right)$ and $U_{q^{-1}}\left(\widehat{\mathfrak{g l}}_{N}\right)$ [20]. It will be shown that one of these morphisms relate pre-BV (3.10) and (3.11). Using second morphism one can obtain dual or left pre-BV in the form of sums over partitions of the Bethe parameters from the formulas for the right pre-BV (3.10) and (3.11).

\subsection{Properties of the $R$-matrix}

Note that the functions (1.3) introduced above, and the modified Izergin determinants (3.3) obey the following relations

$$
\begin{aligned}
\mathrm{g}_{q^{-1}}^{(l)}(v, u)=\mathrm{g}_{q}^{(r)}(u, v), & \mathrm{g}_{q^{-1}}^{(l)}\left(u^{-1}, v^{-1}\right)=\mathrm{g}_{q}^{(r)}(u, v), \\
\mathrm{f}_{q^{-1}}(v, u)=\mathrm{f}_{q}(u, v), & \mathrm{f}_{q^{-1}}\left(u^{-1}, v^{-1}\right)=\mathrm{f}_{q}(u, v), \\
\mathrm{K}_{q^{-1}}^{(l)}(\bar{v} \mid \bar{u})=\mathrm{K}_{q}^{(r)}(\bar{u} \mid \bar{v}), & \mathrm{K}_{q^{-1}}^{(l)}\left(\bar{u}^{-1} \mid \bar{v}^{-1}\right)=\mathrm{K}_{q}^{(r)}(\bar{u} \mid \bar{v}) .
\end{aligned}
$$

We also have the following properties, that can be proved by direct calculation:

$$
\begin{array}{r}
\mathrm{R}_{12}(u, v) \mathrm{R}_{21}(v, u)=\mathrm{f}_{q}(u, v) \mathrm{f}_{q}(v, u) \mathbb{I} \otimes \mathbb{I}, \quad \mathrm{R}_{12}(u, v)^{t_{1} t_{2}}=\mathrm{R}_{21}(u, v), \\
U_{1} U_{2} \mathrm{R}_{12}(u, v) U_{1}^{-1} U_{2}^{-1}=\mathrm{R}_{21}(u, v),
\end{array}
$$

where ${ }^{t}$ denotes the transposition $\left(\mathrm{E}_{i j}\right)^{t}=\mathrm{E}_{j i}, \forall i, j$. We have used auxiliary space notations, where the indices on an operator indicate in which space(s) it acts non trivially, and $\mathrm{R}_{21}(u, v)=$ $P_{12} \mathrm{R}_{12}(u, v) P_{12}$ with $P_{12}$ the permutation of the two spaces 1 and 2 .

\subsection{Iso- and anti- morphisms}

As one can easily deduce from the properties of the R-matrix (4.2), there exist the following morphisms from $U_{q}\left(\widehat{\mathfrak{g l}}_{N}\right)$ to $U_{q^{-1}}\left(\widehat{\mathfrak{g l}}_{N}\right)$. 
Proposition 4.1. (i) The map $\varphi$ defined by

$$
\varphi(T(u))=U \tilde{T}^{t}(u) U^{-1},
$$

where $U$ is given in (4.2), defines an isomorphism from $U_{q}\left(\widehat{\mathfrak{g l}}_{N}\right)$ to $U_{q^{-1}}\left(\widehat{\mathfrak{g l}}_{N}\right)$.

(ii) The map $\psi$ given by

$$
\psi(T(u))=\tilde{T}^{t}\left(u^{-1}\right),
$$

defines an anti-isomorphism from $U_{q}\left(\widehat{\mathfrak{g l}}_{N}\right)$ to $U_{q^{-1}}\left(\widehat{\mathfrak{g l}}_{N}\right)$. In 4.3) and (4.4) $T(u) \in$ $U_{q}\left(\widehat{\mathfrak{g l}}_{N}\right)$ and $\tilde{T}(u) \in U_{q^{-1}}\left(\widehat{\mathfrak{g l}}_{N}\right)$, respectively.

Proof. We start with the defining relation of $U_{q}\left(\widehat{\mathfrak{g l}}_{N}\right)$

$$
\mathrm{R}_{12}(u, v ; q) \cdot T_{1}(u) \cdot T_{2}(v)=T_{2}(v) \cdot T_{1}(u) \cdot \mathrm{R}_{12}(u, v ; q),
$$

apply the transpositions in space 1 and then in space 2 , and conjugation by $U_{1} U_{2}$ to get

$$
U_{1} T_{1}^{t}(u) U_{1}^{-1} \cdot U_{2} T_{2}^{t}(v) U_{2}^{-1} \cdot \mathrm{R}_{12}(u, v ; q)=\mathrm{R}_{12}(u, v ; q) \cdot U_{2} T_{2}^{t}(v) U_{2}^{-1} \cdot U_{1} T_{1}^{t}(u) U_{1}^{-1},
$$

where we have used (4.2). Using again (4.2), we can rewrite (4.6) as

$$
\varphi\left(T_{1}(u)\right) \cdot \varphi\left(T_{2}(v)\right) \cdot \mathrm{R}_{21}\left(v, u ; q^{-1}\right)=\mathrm{R}_{21}\left(v, u ; q^{-1}\right) \cdot \varphi\left(T_{2}(v)\right) \cdot \varphi\left(T_{1}(u)\right) .
$$

After relabeling $1 \leftrightarrow 2$ and $u \leftrightarrow v$, one recognizes in (4.7) the defining relations for $U_{q^{-1}}\left(\widehat{\mathfrak{g l}}_{N}\right)$. This proves $(i)$.

Now, starting again from (4.5), and applying transpositions in space 1 and then in space 2, and the transformation $(u, v) \rightarrow\left(u^{-1}, v^{-1}\right)$, we get

$$
T_{1}^{t}\left(u^{-1}\right) \cdot T_{2}^{t}\left(v^{-1}\right) \cdot \mathrm{R}_{21}\left(u^{-1}, v^{-1}\right)=\mathrm{R}_{21}\left(u^{-1}, v^{-1}\right) \cdot T_{2}^{t}\left(v^{-1}\right) \cdot T_{1}^{t}\left(u^{-1}\right) .
$$

Using once more (4.2), it can be rewritten as

$$
\psi\left(T_{1}(u)\right) \cdot \psi\left(T_{2}(v)\right) \cdot \mathrm{R}_{12}\left(u, v ; q^{-1}\right)=\mathrm{R}_{12}\left(u, v ; q^{-1}\right) \cdot \psi\left(T_{2}(v)\right) \cdot \psi\left(T_{1}(u)\right) .
$$

One recognizes in (4.9) the image of defining relations for $U_{q^{-1}}\left(\widehat{\mathfrak{g l}}_{N}\right)$ under an anti-morphism, i.e. $\psi\left(T_{1}(u) \cdot T_{2}(v)\right)=\psi\left(T_{2}(v)\right) \cdot \psi\left(T_{1}(u)\right)$. This proves $(i i)$.

\section{Closed formulas for dual off-shell BV}

Define the following combinations of the monodromy matrix elements

$$
\begin{aligned}
\mathcal{C}^{\bar{n}}\left(\bar{t}_{\bar{n}}\right) & =\sum_{\operatorname{part}} \prod_{k=1}^{N-1} \prod_{i, j \prec i^{\prime}, j^{\prime}} \mathrm{f}\left(\bar{t}_{i^{\prime}, j^{\prime}}^{k}, \bar{t}_{i, j}^{k}\right) \prod_{k=2}^{N-1}\left(\prod_{i, j \prec i^{\prime}, j^{\prime}} \mathrm{f}\left(\bar{t}_{i, j}^{k}, \bar{t}_{i^{\prime}, j^{\prime}}^{k-1}\right) \prod_{i<j} \mathrm{~K}^{(r)}\left(\bar{t}_{i, j}^{k} \mid \bar{t}_{i, j}^{k-1}\right)\right) \\
& \times \prod_{k=2}^{N-1} \prod_{i, j \prec k, k} T_{k, k}\left(\bar{t}_{i, j}^{k}\right) \prod_{N-1 \geq k \geq 1}\left(\prod_{k<j \leq N} T_{j, k}\left(\bar{t}_{k, j-1}^{k}\right)\right),
\end{aligned}
$$




$$
\begin{aligned}
\widehat{\mathcal{C}}^{n}\left(\bar{t}_{\bar{n}}\right) & =\sum_{\text {part }} \prod_{k=1}^{N-1} \prod_{i, j \prec^{t} i^{\prime}, j^{\prime}} \mathrm{f}\left(\bar{t}_{i^{\prime}, j^{\prime}}^{k}, \bar{t}_{i, j}^{k}\right) \prod_{k=2}^{N-1}\left(\prod_{i, j \prec^{t} i^{\prime}, j^{\prime}} \mathrm{f}\left(\bar{t}_{i, j}^{k}, \bar{t}_{i^{\prime}, j^{\prime}}^{k-1}\right) \prod_{i<j} \mathrm{~K}^{(l)}\left(\bar{t}_{i, j}^{k} \mid \bar{t}_{i, j}^{k-1}\right)\right) \\
& \times \prod_{k=1}^{N-2} \prod_{k, k \prec^{t} i, j} T_{k+1, k+1}\left(\bar{t}_{i, j}^{k}\right) \prod_{1 \leq k \leq N-1}\left(\prod_{1 \leq j \leq k} T_{k+1, j}\left(\bar{t}_{j, k}^{k}\right)\right) .
\end{aligned}
$$

We have the following

\section{Proposition 5.1.}

- The morphism $\varphi$ relates the universal off-shell pre-BV

$$
\left.\varphi\left(\widehat{\mathcal{B}}_{q}^{\bar{n}}\left(\bar{t}_{\bar{n}}\right)\right)=\mathcal{B}_{q^{-1}}^{\omega} \overline{\bar{n}}^{\omega} \bar{t}_{\bar{n}}\right)
$$

where $\omega$ maps the sets of Bethe parameters into the fully permuted sets:

$$
\omega: \bar{t}_{\bar{n}} \rightarrow{ }^{\omega} \bar{t}_{\bar{n}}=\left\{t_{1}^{N-1}, \ldots, t_{n_{N-1}}^{N-1} ; t_{1}^{N-2}, \ldots, t_{n_{N-2}}^{N-2} ; \ldots \ldots ; t_{1}^{2}, \ldots, t_{n_{2}}^{2} ; t_{1}^{1}, \ldots, t_{n_{1}}^{1}\right\},
$$

and accordingly for the sets of cardinalities:

$$
\omega: \bar{n} \rightarrow{ }^{\omega} \bar{n}=\left\{n_{N-1}, n_{N-2}, \ldots, n_{2}, n_{1}\right\} .
$$

- The combinations (5.1) and (5.2) are related to the pre-BV (3.10) and (3.11) by the antimorphism $\psi$

$$
\psi\left(\mathcal{B}_{q}^{\bar{n}}\left(\bar{t}_{\bar{n}}\right)\right)=\mathcal{C}_{q^{-1}}^{\bar{n}}\left(\bar{t}_{\bar{n}}^{-1}\right) \quad \text { and } \quad \psi\left(\widehat{\mathcal{B}}_{q}^{\bar{n}}\left(\bar{t}_{\bar{n}}\right)\right)=\widehat{\mathcal{C}}_{q^{-1}}^{\bar{n}}\left(\bar{t}_{\bar{n}}^{-1}\right),
$$

where

$$
\bar{t}_{\bar{n}}^{-1}=\left\{\left(t_{1}^{1}\right)^{-1}, \ldots,\left(t_{n_{1}}^{1}\right)^{-1} ;\left(t_{1}^{2}\right)^{-1}, \ldots,\left(t_{n_{2}}^{2}\right)^{-1} ; \ldots \ldots ;\left(t_{1}^{N-1}\right)^{-1}, \ldots,\left(t_{n_{N-1}}^{N-1}\right)^{-1}\right\}
$$

is the set of the inverses of Bethe parameters.

Proof. We start with the first item. We write expression for pre-BV (3.10) for the algebra $U_{q^{-1}}\left(\widehat{\mathfrak{g l}}_{N}\right)$ and using the sets $\bar{n} \leftrightarrow{ }^{\omega} \bar{n}, \bar{t}_{\bar{n}} \leftrightarrow{ }^{\omega} \bar{t}_{\bar{n}}$ :

$$
\begin{aligned}
& \left.\mathcal{B}_{q^{-1}}^{\omega} \bar{n}^{\omega} \bar{t}_{\bar{n}}\right)=\sum_{\operatorname{part}} \prod_{k=1}^{N-1} \prod_{i, j \prec i^{\prime}, j^{\prime}} \mathrm{f}_{q^{-1}}\left(\bar{t}_{i^{\prime}, j^{\prime}}^{N-k}, \bar{t}_{i, j}^{N-k}\right) \prod_{k=2}^{N-1} \prod_{i, j \prec i^{\prime}, j^{\prime}} \mathrm{f}_{q^{-1}}\left(\bar{t}_{i, j}^{N-k}, \bar{t}_{i^{\prime}, j^{\prime}}^{N-k+1}\right) \\
& \times \prod_{k=2}^{N-1} \prod_{i<j} \mathrm{~K}_{q^{-1}}^{(l)}\left(\bar{t}_{i, j}^{N-k} \mid \bar{t}_{i, j}^{N-k+1}\right) \prod_{1 \leq k \leq N-1}\left(\prod_{N \geq j>k} \tilde{T}_{k, j}\left(\bar{t}_{k, j}^{N-k}\right)\right) \prod_{k=2}^{N-1} \prod_{i, j \prec k, k} \tilde{T}_{k, k}\left(\bar{t}_{i, j}^{N-k}\right) .
\end{aligned}
$$

Here, $\tilde{T}_{i, j}(t)$ are the matrix elements of the $U_{q^{-1}}\left(\widehat{\mathfrak{g l}}_{N}\right)$ monodromy matrix. 
The next step is to use the formulas (4.1) to change the rational functions $\mathrm{f}_{q^{-1}} \rightarrow \mathrm{f}_{q}$ and $\mathrm{K}_{q^{-1}}^{(l)} \rightarrow \mathrm{K}_{q}^{(r)}$, and apply the morphism $\varphi^{-1}$ to obtain, starting from (5.5):

$$
\begin{aligned}
\varphi^{-1}\left(\mathcal{B}_{q^{-1}}^{\omega} \bar{n}^{\omega}\left(\bar{t}_{\bar{n}}\right)\right)=\sum_{\text {part }} & \prod_{k=1}^{N-1} \prod_{i, j \prec i^{\prime}, j^{\prime}} \mathrm{f}_{q}\left(\bar{t}_{i, j}^{N-k}, \bar{t}_{i^{\prime}, j^{\prime}}^{N-k}\right) \\
& \times \prod_{k=2}^{N-1} \prod_{i, j \prec i^{\prime}, j^{\prime}} \mathrm{f}_{q}\left(\bar{t}_{i^{\prime}, j^{\prime}}^{N-k+1}, \bar{t}_{i, j}^{N-k}\right) \\
& \times \prod_{k=2}^{N-1} \prod_{i<j} \mathrm{~K}_{q}^{(r)}\left(\bar{t}_{i, j}^{N-k+1} \mid \bar{t}_{i, j}^{N-k}\right) \\
& \times \prod_{1 \leq k \leq N-1}^{\longrightarrow}\left(\prod_{N \geq j>k} T_{N+1-j, N+1-k}\left(\bar{t}_{k, j-1}^{N-k}\right)\right) \\
& \times \prod_{k=2}^{N-1} \prod_{i, j \prec k, k} T_{N+1-k, N+1-k}\left(\bar{t}_{i, j}^{N-k}\right) .
\end{aligned}
$$

Let us consider the products in each line of (5.6) separately.

- In the product of the first line we renumber the partition (3.5) of the set $\bar{t}_{\bar{n}}$. We introduce new pairs of integers $\hat{\jmath}=N-i$ and $\hat{\imath}=N-j$ which can be also used to numerate the partition (3.5) since it is clear that for any given $k$ which defines the pair $i, j$ the new integers $\hat{\imath}, \hat{\jmath}$ will also satisfy the condition $1 \leq \hat{\imath} \leq N-k \leq \hat{\jmath} \leq N-1$. One can verify that the condition $i, j \prec i^{\prime}, j^{\prime}$ will be reformulated as $\hat{\imath}^{\prime}, \hat{\jmath}^{\prime} \prec^{t} \hat{\imath}, \hat{\jmath}$. Then, after renaming the integers: $k \rightarrow N-k, \hat{\imath}^{\prime} \rightarrow i, \hat{\jmath}^{\prime} \rightarrow j, \hat{\imath} \rightarrow i^{\prime}$ and $\hat{\jmath} \rightarrow j^{\prime}$, one can see that the first product in (5.6) coincides literally with the first product in (3.11)

$$
\prod_{k=1}^{N-1} \prod_{i, j} \mathrm{f}\left(\bar{t}_{i^{\prime}, j^{\prime}}^{k}, \bar{t}_{i, j}^{k}\right) .
$$

- Using the same arguments, the same change of dumb integers and renaming $k \rightarrow N-k+1$, $\hat{\imath}^{\prime} \rightarrow i, \hat{\jmath}^{\prime} \rightarrow j, \hat{\imath} \rightarrow i^{\prime}$ and $\hat{\jmath} \rightarrow j^{\prime}$, we can prove that the second product in (5.6) is equal to

$$
\prod_{k=2}^{N-1} \prod_{i, j} \mathrm{f}\left(\bar{t}_{i, j}^{k}, \bar{t}_{i^{\prime}, j^{\prime}, j^{\prime}}^{k-1}\right) .
$$

- In the third product of (5.6) we change numeration of the partition of the set $\bar{t}^{N-k+1}$ in such a way to obtain the product

$$
\prod_{k=2}^{N-1} \prod_{i<j} \mathrm{~K}^{(r)}\left(\bar{t}_{i, j}^{k} \mid \bar{t}_{i, j}^{k-1}\right) .
$$


- Renaming the partitions $\left\{\bar{t}_{k, j-1}^{N-k}\right\} \rightarrow\left\{\bar{t}_{N-j+1, N-k}^{N-k}\right\}$ and changing the dumb integers $k \rightarrow$ $N-k$ and $j \rightarrow N-j+1$, we obtain for the forth product in (5.6)

$$
\prod_{N-1 \geq k \geq 1}^{\overleftarrow{ }}\left(\prod_{1 \leq j \leq k} T_{j, k+1}\left(\bar{t}_{j, k}^{k}\right)\right)
$$

- Finally in the fifth product of (5.6), we renumber the partition $\left\{\bar{t}_{i, j}^{N-k}\right\} \rightarrow\left\{\bar{t}_{N-j, N-i}^{N-k}\right\}$ and rename the dumb variables $k \rightarrow N-k, N-j \rightarrow i$ and $N-i \rightarrow j$. It leads to the product

$$
\prod_{k=1}^{N-2} \prod_{k, k \prec^{t} i, j} T_{k+1, k+1}\left(\bar{t}_{i, j}^{k}\right) .
$$

This proves the relation (5.3) of the first item of the Proposition.

For the proof of the second item we consider the first relation in (5.4). The second one can be proved analogously. We rewrite again the expression of the pre-BV (3.10) for $U_{q^{-1}}\left(\widehat{\mathfrak{g l}}_{N}\right)$ and $\bar{t}_{\bar{n}} \leftrightarrow\left(\bar{t}_{\bar{n}}\right)^{-1}$

$$
\begin{aligned}
& \mathcal{B}_{q^{-1}}^{\bar{n}}\left(\left(\bar{t}_{\bar{n}}\right)^{-1}\right)=\sum_{\operatorname{part}} \prod_{k=1}^{N-1} \prod_{i, j \prec i^{\prime}, j^{\prime}} \mathrm{f}_{q^{-1}}\left(\left(\bar{t}_{i^{\prime}, j^{\prime}}^{k}\right)^{-1},\left(\bar{t}_{i, j}^{k}\right)^{-1}\right) \prod_{k=2}^{N-1} \prod_{i, j \prec i^{\prime}, j^{\prime}} \mathrm{f}_{q^{-1}}\left(\left(\bar{t}_{i, j}^{k}\right)^{-1},\left(\bar{t}_{i^{\prime}, j^{\prime}}^{k-1}\right)^{-1}\right) \\
& \times \prod_{k=2}^{N-1} \prod_{i<j} \mathrm{~K}_{q^{-1}}^{(l)}\left(\left(\bar{t}_{i, j}^{k}\right)^{-1}\left(\bar{t}_{i, j}^{k-1}\right)^{-1}\right) \prod_{1 \leq k \leq N-1}\left(\overleftarrow{\prod_{N \geq j>k}} \tilde{T}_{k, j}\left(\left(\bar{t}_{k, j-1}^{k}\right)^{-1}\right)\right) \prod_{k=2} \prod_{i, j \prec k, k} \tilde{T}_{k, k}\left(\left(\bar{t}_{i, j}^{k}\right)^{-1}\right) .
\end{aligned}
$$

Here again $\tilde{T}_{i, j}(t)$ are the matrix elements of the $U_{q^{-1}}\left(\widehat{\mathfrak{g l}}_{N}\right)$ monodromy matrix. Now we apply the antimorphism $\psi$ to (5.7), using once more the relation (4.1) to obtain

$$
\begin{aligned}
& \left.\psi\left(\mathcal{B}_{q^{-1}}^{\bar{n}}\left(\left(\bar{t}_{\bar{n}}\right)^{-1}\right)\right)=\sum_{\operatorname{part}} \prod_{k=1}^{N-1} \prod_{i, j \prec i^{\prime}, j^{\prime}} \mathrm{f}_{q}\left(\bar{t}_{i^{\prime}, j^{\prime}}^{k}, \bar{t}_{i, j}^{k}\right) \prod_{k=2}^{N-1} \prod_{i, j \prec i^{\prime}, j^{\prime}} \mathrm{f}_{q}\left(\bar{t}_{i, j}^{k}\right), \bar{t}_{i^{\prime}, j^{\prime}}^{k-1}\right) \\
& \times \prod_{k=2}^{N-1} \prod_{i<j} \mathrm{~K}_{q}^{(r)}\left(\bar{t}_{i, j}^{k} \mid \bar{t}_{i, j}^{k-1}\right) \prod_{k=2}^{N-1} \prod_{i, j \prec k, k} T_{k, k}\left(\bar{t}_{i, j}^{k}\right) \prod_{N-1 \geq k \geq 1}\left(\prod_{k<j \leq N} T_{j, k}\left(\bar{t}_{k, j-1}^{k}\right)\right) .
\end{aligned}
$$

In (5.8) the property of the antimorphism $\psi$ is used to reverse the order in the product of the non-commuting operators. The r.h.s. of (5.8) coincides with the combination (5.1). Thus, the second item of the Proposition is proved.

Repeating arguments used for the proof of the Proposition 5.1, we can deduce from properties of $\mathbb{B}^{\bar{n}}\left(\bar{t}_{\bar{n}}\right)$, similar properties for $\widehat{\mathbb{B}}_{q}^{\bar{n}}\left(\bar{t}_{\bar{n}}\right)$ and $\mathbb{C}_{q}^{\bar{n}}\left(\bar{t}_{\bar{n}}\right)$. As an illustration, we prove the following. 
Proposition 5.2. The combinations of the monodromy matrix elements (5.1) and (5.2) are the dual pre-BV because their left action onto vacuum vector defined by (1.6) produces the dual off-shell $B V$

$$
\mathbb{C}^{\bar{n}}\left(\bar{t}_{\bar{n}}\right)=\langle 0| \mathcal{C}^{\bar{n}}\left(\bar{t}_{\bar{n}}\right)=\langle 0| \widehat{\mathcal{C}}^{n}\left(\bar{t}_{\bar{n}}\right),
$$

which are eigenvectors under the left action of the transfer matrix $\mathcal{T}(t)=\sum_{i=1}^{N} T_{i, i}(t)$ :

$$
\mathbb{C}^{\bar{n}}\left(\bar{t}_{\bar{n}}\right) \tau\left(t ; \bar{t}_{\bar{n}}\right)=\mathbb{C}^{\bar{n}}\left(\bar{t}_{\bar{n}}\right) \mathcal{T}(t),
$$

with eigenvalue

$$
\tau\left(t ; \bar{t}_{\bar{n}}\right)=\sum_{i=1}^{N} \lambda_{i}^{+}(t) \prod_{j=1}^{n_{i-1}} \mathrm{f}\left(t, t_{j}^{i-1}\right) \prod_{j=1}^{n_{i}} \mathrm{f}\left(t_{j}^{i}, t\right),
$$

provided the Bethe equations

$$
\frac{\lambda_{i}\left(t_{j}^{i}\right)}{\lambda_{i+1}\left(t_{j}^{i}\right)}=(-1)^{n_{i}-1} \prod_{\substack{m=1 \\ m \neq j}}^{n_{i}} \frac{\mathrm{f}\left(t_{j}^{i}, t_{m}^{i}\right)}{\mathrm{f}\left(t_{m}^{i}, t_{j}^{i}\right)} \prod_{m=1}^{n_{i-1}} \mathrm{f}\left(t_{j}^{i}, t_{m}^{i-1}\right)^{-1} \prod_{m=1}^{n_{i+1}} \mathrm{f}\left(t_{m}^{i+1}, t_{j}^{i}\right)
$$

are satisfied.

Proof. We start with the relation (2.12), written in the algebra $U_{q^{-1}}\left(\widehat{\mathfrak{g l}}_{N}\right)$ and for the set of parameters $\bar{t}_{\bar{n}}^{-1}$. Then, we apply $\psi^{-1}$ to get (5.9).

For the remaining part of the proposition, we use a result proved in the paper [17]. It states that in $U_{q}\left(\widehat{\mathfrak{g l}}_{N}\right)$, we have

$$
\mathcal{T}(t) \mathbb{B}^{\bar{n}}\left(\bar{t}_{\bar{n}}\right)=\tau\left(t ; \bar{t}_{\bar{n}}\right) \mathbb{B}^{\bar{n}}\left(\bar{t}_{\bar{n}}\right),
$$

if the Bethe equations (5.12) are satisfied. We reformulate this result for $U_{q^{-1}}\left(\widehat{\mathfrak{g l}}_{N}\right)$, in the following form:

$$
\begin{aligned}
\mathcal{T}_{q^{-1}}(t) \mathbb{B}_{q^{-1}}^{\bar{n}}\left(\bar{t}_{\bar{n}}\right) & =\tau_{q^{-1}}\left(t ; \bar{t}_{\bar{n}}\right) \mathbb{B}_{q^{-1}}^{\bar{n}}\left(\bar{t}_{\bar{n}}\right) \\
& +\sum_{i=1}^{N-1} \sum_{j=1}^{n_{i}} \mathcal{O}_{i j}\left\{\prod_{\substack{m=1 \\
m \neq j}}^{n_{i}} \mathrm{f}_{q^{-1}}\left(t_{m}^{i}, t_{j}^{i}\right) \prod_{m=1}^{n_{i-1}} \mathrm{f}_{q^{-1}}\left(t_{j}^{i}, t_{m}^{i-1}\right) \tilde{T}_{i, i}\left(t_{j}^{i}\right)\right. \\
& \left.-(-1)^{n_{i}-1} \prod_{\substack{m=1 \\
m \neq j}}^{n_{i}} \mathrm{f}_{q^{-1}}\left(t_{j}^{i}, t_{m}^{i}\right) \prod_{m=1}^{n_{i+1}} \mathrm{f}_{q^{-1}}\left(t_{m}^{i+1}, t_{j}^{i}\right) \tilde{T}_{i+1, i+1}\left(t_{j}^{i}\right)\right\}|0\rangle,
\end{aligned}
$$

where $\mathcal{T}_{q^{-1}}(t)=\sum_{i=1}^{N} \tilde{T}_{i i}(t)$ is the transfer matrix in $U_{q^{-1}}\left(\widehat{\mathfrak{g l}}_{N}\right)$, and $\mathcal{O}_{i j}$ are some operators, whose explicit form is not needed for our proof.

A direct calculation, using properties (4.1), shows that $\tau_{q^{-1}}\left(t^{-1} ; \bar{t}_{\bar{n}}^{-1}\right)=\tau_{q}\left(t ; \bar{t}_{\bar{n}}\right)$. It is also clear that $\psi^{-1}\left(\mathcal{T}_{q^{-1}}\left(t^{-1}\right)\right)=\mathcal{T}_{q}(t)$. Then, applying $\psi^{-1}$ to (5.14) written using a spectral 
parameter $t^{-1}$ and the sets $\bar{t}_{\bar{n}}^{-1}$, we get in $U_{q}\left(\widehat{\mathfrak{g l}}_{N}\right)$

$$
\begin{aligned}
\mathbb{C}^{\bar{n}}\left(\bar{t}_{\bar{n}}\right) \mathcal{T}(t)= & \mathbb{C}^{\bar{n}}\left(\bar{t}_{\bar{n}}\right) \tau\left(t ; \bar{t}_{\bar{n}}\right) \\
+ & \sum_{i=1}^{N-1} \sum_{j=1}^{n_{i}}\langle 0|\left\{\prod_{\substack{m=1 \\
m \neq j}}^{n_{i}} \mathrm{f}_{q}\left(t_{m}^{i}, t_{j}^{i}\right) \prod_{m=1}^{n_{i-1}} \mathrm{f}_{q}\left(t_{j}^{i}, t_{m}^{i-1}\right) T_{i, i}\left(t_{j}^{i}\right)\right. \\
& \left.-(-1)^{n_{i}-1} \prod_{\substack{m=1 \\
m \neq j}}^{n_{i}} \mathrm{f}_{q}\left(t_{j}^{i}, t_{m}^{i}\right) \prod_{m=1}^{n_{i+1}} \mathrm{f}_{q}\left(t_{m}^{i+1}, t_{j}^{i}\right) T_{i+1, i+1}\left(t_{j}^{i}\right)\right\} \psi^{-1}\left(\mathcal{O}_{i j}\right),
\end{aligned}
$$

where we have used once more the relations (4.1). This ends the proofs.

\subsection{BV for $U_{q}\left(\widehat{\mathfrak{g l}}_{3}\right)$-symmetric integrable models}

In the paper [18] the action of the monodromy matrix elements onto right off-shell Bethe vectors was calculated in the framework of the current approach and the formulas for the dual off-shell BV were announced. Let us verify that the formulas used in [18] can be obtained from the generic formulas proved in the present paper.

In order to rewrite the $U_{q}\left(\widehat{\mathfrak{g l}}_{3}\right)$ off-shell $\mathrm{BV}$ in the form used in our previous papers we rename the sets of Bethe parameters

$$
\bar{t}^{1}, \bar{t}^{2} \rightarrow \bar{u}, \bar{v}
$$

We rename as follows the subsets of these sets

$$
\begin{gathered}
\bar{t}^{1}=\bar{t}_{1,1}^{1} \cup \bar{t}_{1,2}^{1} \rightarrow \bar{u}_{\mathrm{II}} \cup \bar{u}_{\mathrm{I}}=\bar{u}, \\
\vec{t}^{2}=\bar{t}_{1,2}^{2} \cup \bar{t}_{2,2}^{2} \rightarrow \bar{v}_{\mathrm{I}} \cup \bar{v}_{\mathrm{II}}=\bar{v} .
\end{gathered}
$$

With this renaming of the parameters and the partitions formulas (3.10) and (3.11) yield the following expressions for the off-shell BV:

$$
\begin{aligned}
& \mathbb{B}(\bar{u}, \bar{v})=\sum_{\text {part }} \mathrm{K}^{(l)}\left(\bar{v}_{\mathrm{I}} \mid \bar{u}_{\mathrm{I}}\right) \mathrm{f}\left(\bar{u}_{\mathrm{I}}, \bar{u}_{\mathrm{II}}\right) \mathrm{f}\left(\bar{v}_{\mathrm{II}}, \bar{v}_{\mathrm{I}}\right) T_{13}\left(\bar{u}_{\mathrm{I}}\right) T_{12}\left(\bar{u}_{\mathrm{II}}\right) T_{23}\left(\bar{v}_{\mathrm{II}}\right) \lambda_{2}\left(\bar{v}_{\mathrm{I}}\right)|0\rangle, \\
& \mathbb{B}(\bar{u}, \bar{v})=\sum_{\text {part }} \mathrm{K}^{(r)}\left(\bar{v}_{\mathrm{I}} \mid \bar{u}_{\mathrm{I}}\right) \mathrm{f}\left(\bar{u}_{\mathrm{I}}, \bar{u}_{\mathrm{II}}\right) \mathrm{f}\left(\bar{v}_{\mathrm{II}}, \bar{v}_{\mathrm{I}}\right) T_{13}\left(\bar{v}_{\mathrm{I}}\right) T_{23}\left(\bar{v}_{\mathrm{II}}\right) T_{12}\left(\bar{u}_{\mathrm{II}}\right) \lambda_{2}\left(\bar{u}_{\mathrm{I}}\right)|0\rangle,
\end{aligned}
$$

respectively. The second formula in (5.17) coincides literally with the formula (5.1) in [18] after changing overall normalization of the BV

$$
\mathbb{B}(\bar{u}, \bar{v}) \rightarrow \frac{\mathbb{B}(\bar{u}, \bar{v})}{\mathrm{f}(\bar{v}, \bar{u}) \lambda_{2}(\bar{u}) \lambda_{2}(\bar{v})} .
$$

This change of normalization of the BV is useful for the calculation of the action of the monodromy matrix elements onto them because it remove certain poles in the BV and makes 
formulas for this action quite effective (see details in [18]). The first formula in (5.17) yields an alternative expression for the off-shell BV and their equivalence can be proved directly from the RTT commutation relations (1.1) and certain identities for the Izergin determinants.

Analogously, formulas (5.1) and (5.2) yield the following expressions for the dual or left off-shell BV

$$
\begin{aligned}
& \mathbb{C}(\bar{u}, \bar{v})=\sum_{\text {part }} \mathrm{K}^{(r)}\left(\bar{v}_{\mathrm{I}} \mid \bar{u}_{\mathrm{I}}\right) \mathrm{f}\left(\bar{u}_{\mathrm{I}}, \bar{u}_{\mathrm{II}}\right) \mathrm{f}\left(\bar{v}_{\mathrm{II}}, \bar{v}_{\mathrm{I}}\right) \lambda_{2}\left(\bar{v}_{\mathrm{I}}\right)\langle 0| T_{32}\left(\bar{v}_{\mathrm{II}}\right) T_{21}\left(\bar{u}_{\mathrm{II}}\right) T_{31}\left(\bar{u}_{\mathrm{I}}\right), \\
& \mathbb{C}(\bar{u}, \bar{v})=\sum_{\text {part }} \mathrm{K}^{(l)}\left(\bar{v}_{\mathrm{I}} \mid \bar{u}_{\mathrm{I}}\right) \mathrm{f}\left(\bar{u}_{\mathrm{I}}, \bar{u}_{\mathrm{II}}\right) \mathrm{f}\left(\bar{v}_{\mathrm{II}}, \bar{v}_{\mathrm{I}}\right) \lambda_{2}\left(\bar{u}_{\mathrm{I}}\right)\langle 0| T_{21}\left(\bar{u}_{\mathrm{II}}\right) T_{32}\left(\bar{v}_{\mathrm{II}}\right) T_{31}\left(\bar{v}_{\mathrm{I}}\right),
\end{aligned}
$$

and after the same change of the normalization as in (5.18), the second formula in (5.19) coincides literally with formula (5.2) in the paper [18].

\section{Conclusion}

In this paper we have obtained explicit formulas for the right and left (dual) off-shell BV in the form of sums over partitions of the sets of Bethe parameters using morphisms of the algebra $U_{q}\left(\widehat{\mathfrak{g l}}_{N}\right)$. Our starting formulas were presentations of the off-shell BV in terms of sums over permutations in the sets of Bethe parameters obtained previously in [6, 7] by the current approach. Formulas for left or dual off-shell Bethe vectors are necessary to address the problem of calculation of the scalar products of the BV.

In a previous paper [18, we computed the action of the monodromy matrix elements onto nested off-shell $\mathrm{BV}$ in integrable models with $G L(3)$ trigonometric $R$-matrix. The morphisms (4.3) and antimorphism (4.4) introduced in this paper allows one to relate easily different formulas of these actions. For example, one can obtain using antimorphism $\psi$ the left action of the monodromy matrix elements onto dual off-shell BV from the corresponding formulas of the right action onto right off-shell BV and many other useful relations.

\section{Acknowledgements}

We warmly thank S. Belliard for his contribution at the early stage of this work. Work of S.P. was supported in part by RFBR grant 11-01-00980-a and grant of Scientific Foundation of NRU HSE 12-09-0064. E.R. was supported by ANR Project DIADEMS (Programme Blanc ANR SIMI1 2010-BLAN-0120-02). N.A.S. was supported by the Program of RAS Basic Problems of the Nonlinear Dynamics, RFBR-11-01-00440-a, RFBR-13-01-12405-ofi-m2, SS-4612.2012.1.

\section{References}

[1] P. P. Kulish, N. Yu. Reshetikhin, Diagonalization of $G L(N)$ invariant transfer matrices and quantum $N$-wave system (Lee model), J. Phys. A: 16 (1983) L591-L596. 
[2] P. P. Kulish, N. Yu. Reshetikhin, Generalized Heisenberg ferromagnet and the Gross-Neveu model, Zh. Eksp. Theor. Fiz. 80 (1981) 214-228; Sov. Phys. JETP, 53:1 (1981) 108-114 (Engl. transl.)

[3] P. P. Kulish, N. Yu. Reshetikhin, GL(3)-invariant solutions of the Yang-Baxter equation and associated quantum systems, Zap. Nauchn. Sem. POMI. 120 (1982) 92-121; J. Sov. Math., 34:5 (1982) 1948-1971 (Engl. transl.)

[4] V. Tarasov, A. Varchenko, Jackson integral representations of solutions of the quantized Knizhnik-Zamolodchikov equation, Algebra and Analysis, 6:2 (1994) 90-137; St. Petersburg Math. J. 6:2 (1995) 275-313 (Engl. transl.), arXiv:hep-th/9311040.

[5] V. Tarasov, A. Varchenko, Combinatorial formulae for nested Bethe vectors, SIGMA 9 (2013) 048, arXiv:math/0702277 [math.QA].

[6] S. Khoroshkin, S. Pakuliak, A computation of an universal weight function for the quantum affine algebra $U_{q}\left(\widehat{\mathfrak{g l}}_{N}\right)$, J. of Mathematics of Kyoto University, 48 n.2 (2008) 277-321.

[7] A. Os'kin, S. Pakuliak, A. Silantyev, On the universal weight function for the quantum affine algebra $U_{q}\left(\widehat{\mathfrak{g l}}_{N}\right)$, Algebra and Analysis 21 n.4 (2009) 196-240.

[8] B. Enriquez, V. Rubtsov, Quasi-Hopf algebras associated with $\mathfrak{s l}_{2}$ and complex curves, Israel J. Math 112 (1999) 61-108.

[9] B. Enriquez, S. Khoroshkin, S. Pakuliak, Weight functions and Drinfeld currents, Comm. Math. Phys. 276 (2007) 691-725.

[10] Khoroshkin S., Pakuliak S. Weight function for $U_{q}\left(\widehat{\mathfrak{s l}}_{3}\right)$. Theor. and Math. Phys. 145 n.1 (2005) 1373-1399.

[11] S. Belliard, S. Pakuliak, E. Ragoucy, N. A. Slavnov, Bethe vectors of GL(3)-invariant integrable models, J. Stat. Mech. 1302 (2013) P02020, arXiv:1210.0768.

[12] A. G. Izergin, Partition function of the six-vertex model in a finite volume, Dokl. Akad. Nauk SSSR 297 (1987) 331-333; Sov. Phys. Dokl. 32 (1987) 878-879 (Engl. transl.).

[13] V.E. Korepin, Calculation of norms of Bethe wave functions, Comm. Math. Phys. 86 (1982) 391-418.

[14] J. M. Maillet, V. Terras, On the quantum inverse scattering problem, Nucl. Phys. B 575 (2000) 627-644, hep-th/9911030.

[15] N. Kitanine, J. M. Maillet, V. Terras, Form factors of the $X X Z$ Heisenberg spin-1/2 finite chain, Nucl. Phys. B 554 (1999) 647-678, arXiv:math-ph/9807020.

[16] S. Belliard, S. Pakuliak, E. Ragoucy, N. A. Slavnov, Form factors in SU(3)-invariant integrable models, J. Stat. Mech. 1309 (2013) P04033, arXiv:1211.3968.

[17] L. Frappat, S. Khoroshkin, S. Pakuliak and E. Ragoucy, Bethe Ansatz for the Universal Weight Function, Ann. Henri Poincaré 10 (2009) 513-548, arXiv:0810.3135. 
[18] S. Belliard, S. Pakuliak, E. Ragoucy, N. A. Slavnov, Bethe vectors of quantum integrable models with $G L(3)$ trigonometric R-matrix, SIGMA 9 (2013) 058, arXiv:1304.7602.

[19] S. Belliard, S. Pakuliak, E. Ragoucy, N.A. Slavnov, Highest coefficient of scalar products in SU(3)-invariant models, J. Stat. Mech. (2012) P09003, arXiv:1206.4931.

[20] V. Chari, A. Pressley. A guide to quantum groups. Cambridge University Press, 1994. 